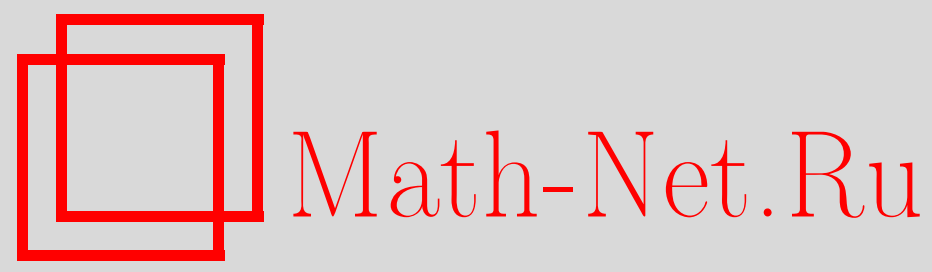

Г. Г. Лаптев, Об отсутствии решений эллиптических дифференциальных неравенств в конических областях, Матем. заметки, 2002, том 71, выпуск 6, 855-866

DOI: https://doi.org/10.4213/mzm390

Использование Общероссийского математического портала Math-Net.Ru подразумевает, что вы прочитали и согласны с пользовательским соглашением http://www . mathnet.ru/rus/agreement

Параметры загрузки:

IP: 54.205 .225 .156

26 апреля 2023 г., 15:54:26

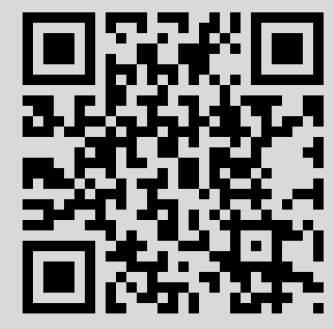




\section{ОБ ОТСУТСТВИИ РЕШЕНИЙ \\ ЭЛЛИПТИЧЕСКИХ ДИФФЕРЕНЦИАЛЬНЫХ НЕРАВЕНСТВ В КОНИЧЕСКИХ ОБЛАСТЯХ}

\section{Г.Г. Лаптев}

Исследуются вопросы отсутствия решений полулинейных эллиптических дифференциальных неравенств и систем второго порядка в конических областях. Доказательство основано на разработанном Митидиери и Похожаевым методе пробных функций без использования теорем сравнения и принципа максимума.

Библиография: 24 названия.

Введение. Статья посвящена нахождению условий отсутствия нетривиальных глобальных решений полулинейных дифференциальных неравенств эллиптического типа в конических областях.

Теория линейных краевых задач в конических областях ведет свой отсчет с классической работы Кондратьева [1]. Современное состояние исследований можно найти в книге Назарова и Пламеневского [2] (см. также литературу там). Изучение полулинейных и нелинейных уравнений проводится в основном на основе известных утверждений для соответствующих линейных задач. При этом получают достаточно тонкие оценки на рост решений [3]-[6]. Следствием этих оценок является, в частности, отсутствие нетривиального решения при некоторых дополнительных условиях.

В настоящей работе для доказательства отсутствия решения применяется теория нелинейной емкости (Похожаев [7]) в виде метода пробных функций, разработанного для эллиптических задач во всем пространстве в статьях Митидиери и Похожаева [8], [9] (см. также работы Курты [10]-[12]).

Отметим, что метод не использует каких-либо сведений из линейной теории и позволяет изучать также разнообразные типы эволюционных (параболических и гиперболических) задач. Заинтересованный читатель может найти соответствующие утверждения в книге Митидиери и Похожаева [13].

Пусть $K_{\omega}$ - область на единичной сфере $S^{N-1} \subset \mathbb{R}^{N}, N \geqslant 3$, с достаточно гладкой гранищей $\partial K_{\omega}$. Конусом $K$ назьвается множество

$$
K=\left\{x=x(r, \omega): 0<r<+\infty, \omega \in K_{\omega}\right\},
$$

где $(r, \omega)$ - сферические координаты точки $x$ в $\mathbb{R}^{N}$. Боковую поверхность конуса обозначаем $\partial K$. Под конической областью $K_{R}$ понимается область $\{x \in K:|x|>R\}$ с полной гранищей $\partial K_{R}$.

Работа вьполнена при финансовой поддержке фонда INTAS, грант № 00-136, программы "Ведушие научные школы”, грант № 00-15-96047, и Российского фонда фундаментальных исследований, грант № 01-01-00884. 
Пусть $\Omega$ - неограниченная область с кусочно-гладкой границей. Далее используются пространства Соболева $W_{q}^{1}(\Omega), W_{q}^{2}(\Omega)$, а также локальное пространство $L_{q, \text { loc }}(\Omega)$, элементы которого принадлежат $L_{q}\left(\Omega^{\prime}\right)$ для любого компактного подмножества $\Omega^{\prime}$ такого, что $\overline{\Omega^{\prime}} \subset \Omega$. Через $C(\bar{\Omega})$ обозначается пространство непрерьвных функций, через $C^{m}(\bar{\Omega})$ - пространство гладких функций. Символ $\Delta$ обозначает оператор Лапласа, $\operatorname{div}=\sum_{i=1}^{N} \partial / \partial x_{i}$, вектор из частных производных обозначаем $D u=\left(\partial u / \partial x_{i}\right)$. Для двух дифференцируемых функций $u(x)$ и $\varphi(x)$ полагаем

$$
D u D \varphi=\sum_{i=1}^{N} \frac{\partial u}{\partial x_{i}} \frac{\partial \varphi}{\partial x_{i}}
$$

Выражение $\int_{\partial K_{R}}(\partial u / \partial n) d x$ обозначает интеграл от производной функции $u$ по направлению внешней нормали $n$ к границе конуса $\partial K_{R}$. Через $c$ и $C$ с индексами будем обозначать постоянные.

Напомним, что оператор Лапласа $\Delta$ в сферических координатах $(r, \omega)$ имеет вид

$$
\Delta=\frac{1}{r^{N-1}} \frac{\partial}{\partial r}\left(r^{N-1} \frac{\partial}{\partial r}\right)+\frac{1}{r^{2}} \Delta_{\omega}=\frac{\partial^{2}}{\partial r^{2}}+\frac{N-1}{r} \frac{\partial}{\partial r}+\frac{1}{r^{2}} \Delta_{\omega}
$$

где $\Delta_{\omega}$ - оператор Бельтрами-Лапласа на единичной сфере $S^{N-1} \subset \mathbb{R}^{N}$.

В дальнейшем постоянно используются наименьшее (первое) собственное значение $\lambda_{\omega} \equiv \lambda_{1}\left(K_{\omega}\right)>0$ и соответствующая собственная функция $\Phi(\omega)$ оператора $\Delta_{\omega}$, являюшиеся решением задачи

$$
\left\{\begin{array}{l}
\Delta_{\omega} \Phi+\lambda \Phi=0 \quad \text { в } \quad K_{\omega} \\
\left.\Phi\right|_{\partial K_{\omega}}=0
\end{array}\right.
$$

Хорошо известно, что $\Phi(\omega)>0$ для $\omega \in K_{\omega}$. Предполагается, что $\Phi(\omega) \leqslant 1$.

1. Модельная задача. Пусть $R$ - фиксированное число. Рассмотрим проблему отсутствия слабых решений задачи

$$
\begin{cases}-\Delta u \geqslant u^{q} \quad \text { в } K_{R} \\ u \geqslant 0, & u \neq \equiv 0\end{cases}
$$

Данная задача для уравнения впервые рассмотрена в работах Бендл и Эссена [14], Бендл [15] и Эгнелла [3]. С использованием предлагаемого метода задача во всем конусе $K$ изучалась в статье автора [16]; утверждения для общих эволюционных неравенств высокого порядка анонсированы в [17]. В работе Бидо-Верон и Похожаева [18] с использованием метода пробных функций рассмотрены более сложные квазилинейные неравенства (с $p$-лапласианом в главной части) в полупространстве, однако с использованием теорем сравнения.

Далее слабое решение будет пониматься в следуюшем смысле.

ОПрЕДЕЛЕНИЕ 1 . Пусть $u(x) \in C\left(\bar{K}_{R}\right)$. Неотрицательная функция $u(x)$ назьвается слабым решением задачи (1), если для любой неотрицательной пробной функции $\varphi(x) \in W_{\infty}^{2}\left(K_{R}\right)$ такой, что $\left.\varphi\right|_{\partial K_{R}}=0$, и финитной по переменной $r$, выполнено интегральное неравенство

$$
\int_{\partial K_{R}} u \frac{\partial \varphi}{\partial n} d x-\int_{K_{R}} u \Delta \varphi d x \geqslant \int_{K_{R}} u^{q} \varphi d x
$$


(предполагается, что все входящие в это неравенство интегралы имеют смысл).

Приведем пример решения задачи (1).

Рассмотрим функцию

$$
u(r, \omega)=\varepsilon r^{s} \Phi(\omega),
$$

где $\Phi(x)$ - введенная вьше собственная функция оператора Бельтрами-Лапласа, $\varepsilon>0$. Используя выражения для оператора Лапласа в сферических координатах и учитывая, что $-\Delta_{\omega} \Phi=\lambda_{\omega} \Phi$, получим

$$
\begin{aligned}
-\Delta u & =-\varepsilon\left(s(s-1) r^{s-2} \Phi(\omega)+s \frac{N-1}{r} r^{s-1} \Phi(\omega)+\frac{r^{s}}{r^{2}} \Delta_{\omega} \Phi\right) \\
& =-\varepsilon \Phi r^{s-2}\left\{s^{2}+s(N-2)-\lambda_{\omega}\right\} .
\end{aligned}
$$

Рассмотрим выражение в фигурных скобках. Введем обозначения для его корней

$$
-s^{*}=-\frac{N-2}{2}-\sqrt{\left(\frac{N-2}{2}\right)^{2}+\lambda_{\omega}}<0, \quad s_{*}=-\frac{N-2}{2}+\sqrt{\left(\frac{N-2}{2}\right)^{2}+\lambda_{\omega}}>0 .
$$

Рассматриваемое выражение меньше нуля, если

$$
-s^{*}<s<s_{*}
$$

Пусть $s^{2}+s(N-2)-\lambda_{\omega}=-\delta<0$. Выберем $s-2=s q$ и $\varepsilon \delta=\varepsilon^{q}$. Тогда

$$
-\varepsilon \Phi r^{s-2}(-\delta)=\varepsilon \delta \Phi r^{s q}=\left(\varepsilon r^{s}\right)^{q} \Phi .
$$

Поскольку мы предполагаем $\Phi \leqslant 1$, то $\Phi \geqslant \Phi^{q}$ и последнее выражение оценивается так:

$$
-\Delta u=\left(\varepsilon r^{s}\right)^{q} \Phi \geqslant\left(\varepsilon r^{s} \Phi\right)^{q}=u^{q} .
$$

Заметим, что последнее неравенство получено при двух условиях на параметр $s$ :

$$
\left\{\begin{array}{l}
-s^{*}<s<s_{*} \\
s-2=s q
\end{array}\right.
$$

откуда следует

$$
\left\{\begin{array}{l}
-s^{*}<-\frac{2}{q-1}<s_{*}, \\
s=-\frac{2}{q-1} .
\end{array}\right.
$$

Поскольку мы интересуемся случаем $q>1$, то в силу $s_{*}>0$ всегда выполнено неравенство $-2 /(q-1)<s_{*}$. Остается только условие

$$
-s^{*}<-\frac{2}{q-1}
$$

откуда

$$
q>1+\frac{2}{s^{*}}=q^{*}
$$

Итак, при $q>q^{*}$ введенная функция $u(x)$ является решением задачи $(1)$. 
Теорема 1. При $1<q \leqslant q^{*}=1+2 / s^{*}$ задача (1) не имеет решения.

Как отмечалось вьше, в используемом методе главным является подходящий выбор пробньгх функций. Введем функцию в $K_{R}$

$$
\xi(x) \equiv \xi(r, \omega)=\left(r^{s_{*}}-R^{s_{*}}\right) \Phi(\omega),
$$

где $s_{*}>0$ определено формулой (2). Очевидно, $\xi(R, \omega)=0, \partial \xi / \partial r(R, \omega) \geqslant 0$ и почти всюду в $K_{R}$

$$
\Delta \xi=\frac{\partial^{2} \xi}{\partial r^{2}}+\frac{N-1}{r} \frac{\partial \xi}{\partial r}-\frac{\lambda_{\omega} \xi}{r^{2}}=R^{s_{*}} \frac{\lambda_{\omega}}{r^{2}} \Phi(\omega) \geqslant 0 .
$$

Пробную функцию будем брать в виде произведения

$$
\varphi_{\rho}(x)=\eta_{\rho}(x) \xi(x) .
$$

Здесь $\eta_{\rho}(x) \equiv \eta_{\rho}(|x|)=\eta_{\rho}(r) \in C^{2}[0, \infty)$ - стандартная срезающая функция такая, что $\eta_{\rho}(r)=1$ для $0 \leqslant r \leqslant \rho, \eta_{\rho}(r) \equiv 0$ для $r>2 \rho$ и при достаточно больших $\rho$

$$
\frac{\eta_{\rho}^{p}}{\eta_{\rho}^{p-1}}=\eta_{\rho} \leqslant c_{\eta}, \quad \frac{\left|d \eta_{\rho} / d r\right|^{p}}{\eta_{\rho}^{p-1}} \leqslant \frac{c_{\eta}}{\rho^{p}}, \quad \frac{\left|\Delta \eta_{\rho}\right|^{p}}{\eta_{\rho}^{p-1}} \leqslant \frac{c_{\eta}}{\rho^{2 p}},
$$

где $c_{\eta}$ не зависит от $\rho$. Возможность построения такой функции $\eta_{\rho}$ известна из работ [8]-[10].

Очевидны также неравенства (при $\rho \geqslant 2 R$ и $\rho \leqslant|x| \leqslant 2 \rho$ )

$$
\begin{gathered}
c_{\xi 0} \Phi(\omega) \rho^{s_{*}} \leqslant \xi(r, \omega) \leqslant c_{\xi 1} \Phi(\omega) \rho^{s_{*}}, \\
\left|\frac{\partial \xi}{\partial r}(r, \omega)\right| \leqslant c_{\xi 1} \Phi(\omega) \rho^{s_{*}-1}, \quad|\Delta \xi| \leqslant c_{\xi 1} \Phi(\omega) \rho^{s_{*}-2},
\end{gathered}
$$

где $c_{\xi 0}, c_{\xi 1}$ зависят от $R$, но не зависят от $\rho$ и $r$.

$\mathrm{B}$ дальнейшем нам понадобится оценка интеграла

$$
\int_{K_{R} \cap\{\rho<|x|<2 \rho\}} \frac{\left|\Delta \varphi_{\rho}\right|^{p}}{\varphi_{\rho}^{p-1}} d x
$$

при $p>1$, зависяшая от $\rho$. Для ее получения воспользуемся приведенными вьше неравенствами и известной формулой

$$
\Delta \varphi_{\rho}=\Delta\left(\eta_{\rho} \xi\right)=\Delta \eta_{\rho} \xi+2\left(\nabla \eta_{\rho}, \nabla \xi\right)+\eta_{\rho} \Delta \xi=\Delta \eta_{\rho} \xi+2 \frac{d \eta_{\rho}}{d r} \frac{\partial \xi}{\partial r}+\eta_{\rho} \Delta \xi .
$$

Тогда в области $\rho<|x|<2 \rho$ выполняется неравенство

$$
\begin{aligned}
\frac{\left|\Delta \varphi_{\rho}\right|^{p}}{\varphi_{\rho}^{p-1}}=\frac{\left|\Delta\left(\eta_{\rho} \xi\right)\right|^{p}}{\eta_{\rho}^{p-1} \xi^{p-1}} & \leqslant c_{p} \frac{\left|\Delta \eta_{\rho}\right|^{p}}{\eta_{\rho}^{p-1}} \frac{\xi^{p}}{\xi^{p-1}}+c_{p} \frac{\left|d \eta_{\rho} / d r\right|^{p}}{\eta_{\rho}^{p-1}} \frac{|\partial \xi / \partial r|^{p}}{\xi^{p-1}}+c_{p} \frac{\left|\eta_{\rho}\right|^{p}}{\eta_{\rho}^{p-1}} \frac{|\Delta \xi|^{p}}{\xi^{p-1}} \\
& \leqslant c_{p} c_{\eta} \frac{c_{\xi 1}^{p}}{c_{\xi 0}^{p-1}} \Phi(\omega) \rho^{s_{*}-2 p}
\end{aligned}
$$

откуда для искомого интеграла получаем

$$
\begin{aligned}
\int_{K_{R} \cap\{\rho<|x|<2 \rho\}} \frac{\left|\Delta \varphi_{\rho}\right|^{p}}{\varphi_{\rho}^{p-1}} d x & \leqslant c_{p} c_{\eta} \frac{c_{\xi 1}^{p}}{c_{\xi 0}^{p-1} \rho^{s_{*}-2 p}} \int_{\rho}^{2 \rho} r^{N-1} d r \int_{K_{\omega}} \Phi(\omega) d \omega \\
& \leqslant c_{N p \eta \xi} \rho^{-2 p+s_{*}+N},
\end{aligned}
$$

где постоянная $c_{N p \eta \xi}$ не зависит от $\rho$. 
ДокАЗАТЕЛЬСтво теоРемЫ 1 . Доказьваем от противного. Пусть $u(x)-$ решение задачи (1) с $1<q \leqslant q^{*}$. Наша цель-показать, что тог да $u \equiv 0$. Согласно определению 1 с пробной функцией $\varphi(x)=\varphi_{\rho}(x)$ (где взято $p=q^{\prime}>1$ ) это означает

$$
\begin{aligned}
\int_{K_{R}} u^{q} \varphi_{\rho} d x \leqslant & \int_{\partial K_{R}} u \frac{\partial \varphi_{\rho}}{\partial n} d x-\int_{K_{R}} u \Delta \varphi_{\rho} d x \\
= & -\int_{\{|x|=R\} \cap \partial K_{R}} u \frac{\partial \varphi_{\rho}}{\partial r} d x+\int_{\{|x|>R\} \cap \partial K_{R}} u \frac{\partial \varphi_{\rho}}{\partial n} d x \\
& -\int_{K_{R} \cap\{\rho<|x|<2 \rho\}} u \Delta \varphi_{\rho} d x-\int_{K_{R} \cap\{|x|<\rho\}} u \Delta \varphi_{\rho} d x .
\end{aligned}
$$

Рассмотрим интегралы в правой части. По построению имеем

$$
\left.\frac{\partial \varphi_{\rho}}{\partial r}\right|_{r=R}=\left.\frac{\partial \xi}{\partial r}\right|_{r=R} \geqslant 0
$$

поэтому первьй интеграл неотрицателен. Далее заметим, что мы предполагаем $u \geqslant 0$ в $K_{R}$, откуда в силу неравенства $\Delta \xi \geqslant 0$ заключаем, что последний интеграл также неотрицателен (без учета знака). Наконец, на боковой поверхности конуса $K_{R}$ имеем

$$
\frac{\partial \varphi_{\rho}}{\partial n}=\eta_{\rho}(r)\left(r^{s_{*}}-R^{s_{*}}\right) \frac{\partial \Phi(\omega)}{\partial n_{\omega}} \leqslant 0
$$

где $n_{\omega}$ - внешняя нормаль к гранище области $K_{\omega}$. Вывод о неположительности производной $\partial \Phi(\omega) / \partial n_{\omega}$ на границе области $K_{\omega}$ сделан на том основании, что $\Phi(w) \geqslant 0$ в $K_{\omega}$ и $\left.\Phi(\omega)\right|_{\partial K_{\omega}}=0$ по построению. Таким образом, и второй интеграл в правой части неравенства (6) неположителен. В совокупности из приведенных рассуждений следует, что

$$
\int_{K_{R}} u^{q} \varphi_{\rho} d x \leqslant \int_{K_{R} \cap\{\rho<|x|<2 \rho\}} u\left|\Delta \varphi_{\rho}\right| d x .
$$

Для оценки правой части этого неравенства применим неравенство Гёльдера. Получим

$$
\begin{aligned}
\int_{K_{R}} u^{q} \varphi_{\rho} d x & =\int_{K_{R} \cap\{\rho<|x|<2 \rho\}} u^{q} \varphi_{\rho} d x+\int_{K_{R} \cap\{|x|<\rho\}} u^{q} \xi d x \\
& \leqslant \int_{K_{R} \cap\{\rho<|x|<2 \rho\}} u\left|\Delta \varphi_{\rho}\right| d x \\
& \leqslant\left(\int_{K_{R} \cap\{\rho<|x|<2 \rho\}} u^{q} \varphi_{\rho} d x\right)^{1 / q}\left(\int_{K_{R} \cap\{\rho<|x|<2 \rho\}} \frac{\left|\Delta \varphi_{\rho}\right|^{q^{\prime}}}{\varphi_{\rho}^{q^{\prime}-1}} d x\right)^{1 / q^{\prime}},
\end{aligned}
$$

откуда с учетом оценки (5) для последнего интеграла справа будем иметь

$$
\int_{K_{R} \cap\{|x|<\rho\}} u^{q} \xi d x \leqslant \int_{K_{R} \cap\{\rho<|x|<2 \rho\}} \frac{\left|\Delta \varphi_{\rho}\right|^{q^{\prime}}}{\varphi_{\rho}^{q^{\prime}-1}} d x \leqslant c_{0} \rho^{-2 q^{\prime}+s_{*}+N} .
$$

Так как подынтегральное выражение в левой части не зависит от $\rho$, можем перейти к пределу по $\rho \rightarrow 0$. В случае

$$
-2 q^{\prime}+s_{*}+N \leqslant 0
$$


это приводит к соотношению

$$
\int_{K_{R}} u^{q} \xi d x \leqslant c_{0}
$$

Тогда в силу равностепенной непрерьвности интеграла и ввиду оценки $\varphi_{\rho} \leqslant \xi$ имеем

$$
\int_{K_{R} \cap\{\rho<|x|<2 \rho\}} u^{q} \varphi_{\rho} d x \leqslant \int_{K_{R} \cap\{\rho<|x|<2 \rho\}} u^{q} \xi d x=\varepsilon(\rho) \rightarrow 0 \quad \text { при } \quad \rho \rightarrow 0 .
$$

Возвращаясь теперь к неравенству (7), получим

$$
\int_{K_{R} \cap\{|x|<\rho\}} u^{q} \xi d x \leqslant \varepsilon^{1 / q}(\rho) c_{0}^{1 / q^{\prime}} \rightarrow 0
$$

при $\rho \rightarrow 0$, т.е. в пределе

$$
\int_{K_{R}} u^{q} \xi d x=0
$$

откуда в силу положительности $\xi$ в $K_{R}$ следует $u \equiv 0$, что противоречит нашему предположению о существовании нетривиального решения (техника исследования критического случая $-2 q^{\prime}+s_{*}+N=0$ заимствована из работ [8], [9]).

Элементарными преобразованиями из неравенства

$$
-2 q^{\prime}+s_{*}+N \leqslant 0
$$

получаем условие отсутствия нетривиального решения: $1<q \leqslant q^{*}$.

Приведенное определение обобшенного решения может быть расширено, в частности, рассмотрением пространства суммируемых функций $L_{q}\left(K_{R}\right)$ вместо пространства непрерьвных функций.

2. Система неравенств. Рассмотрим систему

$$
\begin{cases}-\operatorname{div}\left(|x|^{\alpha_{1}} D u\right) \geqslant|x|^{\beta_{1}} v^{q_{1}}, & \beta_{1}-\alpha_{1}+2>0, \\ -\operatorname{div}\left(|x|^{\alpha_{2}} D v\right) \geqslant|x|^{\beta_{2}} u^{q_{2}}, & \beta_{2}-\alpha_{2}+2>0, \quad \text { в } K_{R} . \\ u \geqslant 0, \quad u \neq 0, & v \geqslant 0, \quad v \not \equiv 0,\end{cases}
$$

Для случая всего пространства такого рода системы рассмотрены в книге [13] (даже без предположения о положительности решения) и работе [19]. Результаты об отсутствии решений более простьх систем в полном конусе получены в работе автора [16]. Заметим, что полученные ниже результаты можно переформулировать (с использованием преобразования Кельвина) также для задач с сильно вырождаюшимися коэффициентами в ограниченной области и получить на этом пути аналоги ряда результатов Брезиса и Кабре [20], Галахова [21], Похожаева и Тесея [22] (см. также [23]) для областей с коническими точками границы. 
ОПРЕДЕЛЕНИЕ 2. Пара функций $u, v \in C\left(K_{R}\right)$ назьвается слабым решением задачи (9), если для любых неотрицательных пробных функций $\varphi_{1}(x), \varphi_{2}(x) \in W_{\infty}^{2}\left(K_{R}\right)$ таких, что $\left.\varphi_{1}\right|_{\partial K_{R}}=0,\left.\varphi_{2}\right|_{\partial K_{R}}=0$, и финитных по переменной $r$, выполнены интегральные неравенства

$$
\left\{\begin{array}{l}
\int_{\partial K_{R}} u|x|^{\alpha_{1}} \frac{\partial \varphi_{1}}{\partial n} d x-\int_{K_{R}} u \operatorname{div}\left(|x|^{\alpha_{1}} D \varphi_{1}\right) d x \geqslant \int_{K_{R}}|x|^{\beta_{1}} v^{q_{1}} \varphi_{1} d x \\
\int_{\partial K_{R}} v|x|^{\alpha_{2}} \frac{\partial \varphi_{2}}{\partial n} d x-\int_{K_{R}} v \operatorname{div}\left(|x|^{\alpha_{2}} D \varphi_{2}\right) d x \geqslant \int_{K_{R}}|x|^{\beta_{2}} u^{q_{2}} \varphi_{2} d x
\end{array}\right.
$$

(предполагается, что все интегралы имеют смысл).

Введем параметры

$$
\sigma_{1}=2+\beta_{1}-\alpha_{1}+s_{\alpha_{1}}^{*}, \quad \sigma_{2}=2+\beta_{2}-\alpha_{2}+s_{\alpha_{2}}^{*}
$$

ТЕОрема 2. Задача (9) не имеет глобального неотрицательного нетривиального слабого решения, если

$$
\max \left\{k_{1}, k_{2}\right\} \geqslant 0
$$

$2 \partial e$

$k_{1}=\left(\sigma_{1}-s_{\alpha_{2}}^{*}\right) q_{1}-\left(\sigma_{2}-\beta_{2}+\beta_{1}\right)\left(q_{1}-1\right)+\left(\sigma_{2}-s_{\alpha_{1}}^{*}\right) q_{2} q_{1}-\left(\sigma_{1}-\beta_{1}+\beta_{2}\right)\left(q_{2}-1\right) q_{1}$, $k_{2}=\left(\sigma_{2}-s_{\alpha_{1}}^{*}\right) q_{2}-\left(\sigma_{1}-\beta_{1}+\beta_{2}\right)\left(q_{2}-1\right)+\left(\sigma_{1}-s_{\alpha_{2}}^{*}\right) q_{1} q_{2}-\left(\sigma_{2}-\beta_{2}+\beta_{1}\right)\left(q_{1}-1\right) q_{2}$, $s_{\alpha_{1}}^{*}, s_{\alpha_{2}}^{*}$ определяются формулой (11).

ДокАЗАТЕЛЬСтво. Пусть $u, v$ - слабое решение задачи (9). Для упрощения последующих выкладок введем оператор

$$
A_{\alpha} \varphi=\operatorname{div}\left(|x|^{\alpha} D \varphi\right) .
$$

При $\alpha=0$ имеем $A_{0} \varphi=\Delta \varphi$.

Выберем пробные функции $\varphi_{\alpha}(x)=\varphi_{\alpha, \rho}(x)$ по формуле $(4)$, но при определении $\xi$ по формуле (3) и в дальнейших рассуждениях в качестве $s_{*}$ и $s^{*}$ положим

$$
\begin{aligned}
& s_{* \alpha}=-\frac{\alpha+N-2}{2}+\sqrt{\left(\frac{\alpha+N-2}{2}\right)^{2}+\lambda_{\omega}}, \\
& s_{\alpha}^{*}=\frac{\alpha+N-2}{2}+\sqrt{\left(\frac{\alpha+N-2}{2}\right)^{2}+\lambda_{\omega}}=s_{* \alpha}+\alpha+N-2,
\end{aligned}
$$

где $\alpha=\alpha_{1}$ или $\alpha=\alpha_{2}$. Перейдя к сферической системе координат, аналогично модельной задаче (см. также [16]) можно убедиться, что

$$
\left\{x \in K_{R}: A_{\alpha} \varphi_{\alpha, \rho}<0\right\} \subseteq K_{R} \cap\{\rho<|x|<2 \rho\}
$$

и справедливы оценки (для $p>1$ )

$$
\int_{K_{R} \cap\{\rho<r<2 \rho\}} \frac{\left|A_{\alpha} \varphi_{\alpha, \rho}\right|^{p}}{|x|^{\beta(p-1)} \varphi_{\gamma, \rho}^{p-1}} d x \leqslant \frac{C}{\rho^{\left(s_{\gamma}^{*}-s_{\alpha}^{*}+2+\beta-\gamma\right) p-s_{\alpha}^{*}+\alpha-\beta-2}} .
$$


Заметим, что здесь введен параметр $\gamma$, в качестве которого в дальнейшем будут выбираться $\alpha_{1}$ или $\alpha_{2}$.

Положим $\varphi_{1}(x)=\varphi_{\alpha_{1}, \rho}(x)$ и $\varphi_{2}(x)=\varphi_{\alpha_{2}, \rho}(x)$. Используя рассуждения из доказательства теоремы 1 о знаке производной по нормали к границе конической области $K_{R}$ функции $\varphi_{\alpha, \rho}(x)$, из определения слабого решения получаем

$$
\left\{\begin{array}{l}
\int_{K_{R}}|x|^{\beta_{1}} v^{q_{1}} \varphi_{1} d x \leqslant-\int_{K_{R}} u A_{\alpha_{1}} \varphi_{1} d x, \\
\int_{K_{R}}|x|^{\beta_{2}} u^{q_{2}} \varphi_{2} d x \leqslant-\int_{K_{R}} v A_{\alpha_{2}} \varphi_{2} d x .
\end{array}\right.
$$

Для оценки интегралов справа применим неравенство Гёльдера:

$$
\begin{aligned}
& \int_{K_{R}}|x|^{\beta_{1}} v^{q_{1}} \varphi_{1} d x \leqslant-\int_{K_{R}} u A_{\alpha_{1}} \varphi_{1} d x \leqslant-\int_{A_{\alpha_{1}} \varphi_{1}<0}|x|^{\beta_{2} / q_{2}} u \varphi_{2}^{1 / q_{2}} \frac{A_{\alpha_{1}} \varphi_{1}}{|x|^{\beta_{2} / q_{2} \varphi_{2}^{1 / q_{2}}} d x} \\
& \leqslant\left(\int_{K_{R} \cap\{\rho<|x|<2 \rho\}}|x|^{\beta_{2}} u^{q_{2}} \varphi_{2} d x\right)^{1 / q_{2}}\left(\int_{K_{R} \cap\{\rho<|x|<2 \rho\}} \frac{\left|A_{\alpha_{1}} \varphi_{1}\right|_{2}^{q_{2}^{\prime}}}{|x|^{\beta_{2}\left(q_{2}^{\prime}-1\right)} \varphi_{2}^{q_{2}^{\prime}-1}} d x\right)^{1 / q_{2}^{\prime}} \\
& =\left(\int_{K_{R} \cap\{\rho<|x|<2 \rho\}}|x|^{\beta_{2}} u^{q_{2}} \varphi_{2} d x\right)^{1 / q_{2}} J_{1}^{1 / q_{2}^{\prime}}, \\
& \int_{K_{R}}|x|^{\beta_{2}} u^{q_{2}} \varphi_{2} d x \leqslant-\int_{K_{R}} v A_{\alpha_{2}} \varphi_{2} d x \leqslant-\int_{A_{\alpha_{2}} \varphi_{2}<0}|x|^{\beta_{1} / q_{1}} v \varphi_{1}^{1 / q_{1}} \frac{A_{\alpha_{2}} \varphi_{2}}{|x|^{\beta_{1} / q_{1} \varphi_{1}^{1 / q_{1}}} d x} \\
& \leqslant\left(\int_{K_{R} \cap\{\rho<|x|<2 \rho\}}|x|^{\beta_{1}} v^{q_{1}} \varphi_{1} d x\right)^{1 / q_{1}}\left(\int_{K_{R} \cap\{\rho<|x|<2 \rho\}} \frac{\left|A_{\alpha_{2}} \varphi_{2}\right|^{q_{1}^{\prime}}}{|x|^{\beta_{1}\left(q_{1}^{\prime}-1\right)} \varphi_{1}^{q_{1}^{\prime}-1}} d x\right)^{1 / q_{1}^{\prime}} \\
& =\left(\int_{K_{R} \cap\{\rho<|x|<2 \rho\}}|x|^{\beta_{1}} v^{q_{1}} \varphi_{1} d x\right)^{1 / q_{1}} J_{2}^{1 / q_{1}^{\prime}},
\end{aligned}
$$

причем согласно (12) имеем оценки

$$
\begin{aligned}
& J_{1}=\int_{K_{R} \cap\{\rho<|x|<2 \rho\}} \frac{\left|A_{\alpha_{1}} \varphi_{1}\right|_{2}^{\prime}}{|x|^{\beta_{2}\left(q_{2}^{\prime}-1\right)} \varphi_{2}^{q_{2}^{\prime}-1}} d x \leqslant \frac{C}{\rho^{\left(s_{\alpha_{2}}^{*}-s_{\alpha_{1}}^{*}+2+\beta_{2}-\alpha_{2}\right) q_{2}^{\prime}-s_{\alpha_{1}}^{*}+\alpha_{1}-\beta_{2}-2}}, \\
& J_{2}=\int_{K_{R} \cap\{\rho<|x|<2 \rho\}} \frac{\left|A_{\alpha_{2}} \varphi_{2}\right|_{1}^{q_{1}^{\prime}}}{|x|^{\beta_{1}\left(q_{1}^{\prime}-1\right)} \varphi_{1}^{q_{1}^{\prime}-1}} d x \leqslant \frac{C}{\rho^{\left(s_{\alpha_{1}}^{*}-s_{\alpha_{2}}^{*}+2+\beta_{1}-\alpha_{1}\right) q_{1}^{\prime}-s_{\alpha_{2}}^{*}+\alpha_{2}-\beta_{1}-2}} .
\end{aligned}
$$

Подставим неравенство (14) в (13). Тогда

$$
\int_{K_{R}}|x|^{\beta_{1}} v^{q_{1}} \varphi_{1} d x \leqslant\left(\int_{K_{R} \cap\{\rho<|x|<2 \rho\}}|x|^{\beta_{1}} v^{q_{1}} \varphi_{1} d x\right)^{1 /\left(q_{1} q_{2}\right)} J_{2}^{1 /\left(q_{1}^{\prime} q_{2}\right)} J_{1}^{1 / q_{2}^{\prime}},
$$

откуда с учетом нашего предположения о нетривиальности $v(x)$ и оценок $(15)$

$$
\int_{K_{R}}|x|^{\beta_{1}} v^{q_{1}} \varphi_{1} d x \leqslant\left(J_{2}^{1 / q_{1}^{\prime}} J_{1}^{q_{2}-1}\right)^{q_{1} /\left(q_{1} q_{2}-1\right)} \leqslant \frac{C}{\rho^{k_{1} /\left(q_{1} q_{2}-1\right)}} .
$$

Аналогично, подставляя (13) в (14), приходим к оценке

$$
\int_{K_{R}}|x|^{\beta_{2}} u^{q_{2}} \varphi_{2} d x \leqslant\left(J_{1}^{1 / q_{2}^{\prime}} J_{2}^{q_{1}-1}\right)^{q_{2} /\left(q_{1} q_{2}-1\right)} \leqslant \frac{C}{\rho^{k_{2} /\left(q_{1} q_{2}-1\right)}} .
$$


Аналогично модельной задаче легко видеть, что если $k_{1} \geqslant 0$, то $v(x) \equiv 0$, если же $k_{2} \geqslant 0$, то $u(x) \equiv 0$. Очевидно, что если хотя бы одна из функций $u(x)$ или $v(x)$ тождественно равна нулю, то равна нулю и другая. Таким образом, условие отсутствия нетривиального решения принимает вид

$$
\max \left\{k_{1}, k_{2}\right\} \geqslant 0 \text {. }
$$

Теорема доказана.

Приведем конкретные примеры использования теоремы 2.

СлЕДСТВИЕ 1. Задача

$$
\left\{\begin{array}{l}
-\operatorname{div}\left(|x|^{\alpha} D u\right) \geqslant|x|^{\beta} u^{q}, \quad \beta-\alpha+2>0, \quad \text { в } \quad K_{R} \\
u \geqslant 0, \quad u \neq 0,
\end{array}\right.
$$

не имеет слабого решения, если

$$
1<q \leqslant q^{*}=1+\frac{2+\beta-\alpha}{s_{\alpha}^{*}} .
$$

СлЕДСТВИЕ 2. Задача

$$
\left\{\begin{array}{l}
-\operatorname{div}\left(|x|^{\alpha} D u\right) \geqslant|x|^{\beta} v^{q_{1}}, \quad \beta-\alpha+2>0, \\
-\operatorname{div}\left(|x|^{\alpha} D v\right) \geqslant|x|^{\beta} u^{q_{2},} \\
u \geqslant 0, \quad u \neq 0, \\
v \geqslant 0, \quad v \not \equiv 0,
\end{array}\right.
$$

не имеет слабого решения, если

$$
\max \left\{\gamma_{1}, \gamma_{2}\right\} \geqslant \frac{s_{\alpha}^{*}}{2+\beta-\alpha}
$$

əde

$$
\gamma_{1}=\frac{q_{1}+1}{q_{2} q_{1}-1}, \quad \gamma_{2}=\frac{q_{2}+1}{q_{2} q_{1}-1}
$$

ЗАмЕчАниЕ 1. Отметим один специальньй частньй случай. Условия $2+\beta_{1}-\alpha_{1}>0$ и $2+\beta_{2}-\alpha_{2}>0$ дают возможность считать параметры $\alpha_{1}$ и $\alpha_{2}$ сколь угодно большими по модулю отрицательными числами. Используем это наблюдение в следующей ситуации.

Полагаем $\alpha_{1}=\beta_{1}=\beta_{2}=0$, оставив только параметр $\alpha_{2} \rightarrow-\infty$, т.е. рассмотрим задачу

$$
\left\{\begin{array}{l}
-\Delta u \geqslant v^{q_{1}} \\
-\operatorname{div}\left(|x|^{\alpha_{2}} D v\right) \geqslant u^{q_{2}}, \quad \alpha_{2}<0, \quad \text { в } K_{R} . \\
u \geqslant 0, \quad u \neq 0 \\
v \geqslant 0, \quad v \neq 0
\end{array}\right.
$$

Вьпишем показатели $\sigma_{1}, \sigma_{2}$ и $k_{1}$ при указанньх условиях:

$$
\begin{gathered}
\sigma_{1}=2+s_{0}^{*}, \quad \sigma_{2}=2-\alpha_{2}+s_{\alpha_{2}}^{*} \\
k_{1}=\left(2+s_{0}^{*}-s_{\alpha_{2}}^{*}\right) q_{1}-\left(2-\alpha_{2}+s_{\alpha_{2}}^{*}\right)\left(q_{1}-1\right) \\
+\left(2-\alpha_{2}+s_{\alpha_{2}}^{*}-s_{0}^{*}\right) q_{1} q_{2}-\left(2+s_{0}^{*}\right) q_{1}\left(q_{2}-1\right)
\end{gathered}
$$


С помощью очевидных преобразований $k_{1}$ условие $k_{1} \geqslant 0$ приведем к виду

$$
k_{1}=q_{1}\left(-\alpha_{2}+2-s_{\alpha_{2}}^{*}+\left(q_{2}-1\right)\left(-\alpha_{2}+s_{\alpha_{2}}^{*}-2 s_{0}\right)\right)-\alpha_{2}+2+s_{\alpha_{2}}^{*} \geqslant 0 .
$$

Легко видеть, что это неравенство будет вьполнено при любых $q_{1}, q_{2}>1$, если

$$
\left\{\begin{array}{l}
-\alpha_{2}+2-s_{\alpha_{2}}^{*} \geqslant 0 \\
-\alpha_{2}+s_{\alpha_{2}}^{*}-2 s_{0} \geqslant 0 \\
-\alpha_{2}+2+s_{\alpha_{2}}^{*} \geqslant 0
\end{array}\right.
$$

По нашему предположению $-\alpha_{2} \rightarrow+\infty$, и из формулы (11) легко видеть, что тогда $s_{\alpha_{2}}^{*} \rightarrow 0$. Таким образом, можно выбрать такое $\alpha_{2}$, чтобы все эти три неравенства удовлетворялись одновременно.

Итак, построен пример задачи с сильной сингулярностью в главной части, которая не имеет глобального нетривиального неотрицательного решения при всех $q_{1}, q_{2}>1$.

3. Отсутствие решений задач из заданного функционального класса: модельная задача. В предыдущих пунктах мы рассматривали вопросы отсутствия решений без каких-либо предположений о поведении решения на бесконечности. Если же потребовать, чтобы решение было суммируемо в некоторой степени $l>1$, то, в свою очередь, возникают определенные критические показатели. В данной постановке во всем пространстве задача рассматривалась в работе [19]. Соответствующие утверждения известны как обобшения теоремы Бернштейна [24].

Проиллюстрируем сказанное на примере задачи (1). Под слабым решением будем понимать функцию $u(x) \in L_{l}\left(K_{R}\right)$, удовлетворяющую определению 1 . Подчеркнем, что здесь мы не имеем в виду локальное пространство $L_{l, l o c}\left(K_{R}\right)$.

Teopema 3. Пусть

$$
1<l \leqslant l^{*}=\frac{N}{s^{*}} .
$$

Тогда задача (1) не имеет слабого решения из $L_{l}\left(K_{R}\right)$ ни при каких $q \in \mathbb{R}$.

ДокАЗАТЕЛЬСтво проводим от противного. Пусть $u \in L_{l}\left(K_{R}\right)$ - слабое (нетривиальное) решение задачи (1). Аналогично доказательству теоремы 1 с соответствующей пробной функцией $\varphi_{\rho}(x)$ из определения слабого решения с использованием неравенства Гёльдера получим

$$
\begin{aligned}
\int_{K_{R}} u^{q} \varphi_{\rho} d x & \leqslant \int_{K_{R} \cap\{\rho<|x|<2 \rho\}} u\left|\Delta \varphi_{\rho}\right| d x \\
& \leqslant\left(\int_{K_{R} \cap\{\rho<|x|<2 \rho\}} u^{l} d x\right)^{1 / l}\left(\int_{K_{R} \cap\{\rho<|x|<2 \rho\}}\left|\Delta \varphi_{\rho}\right|^{l^{\prime}} d x\right)^{1 / l^{\prime}} \\
& \leqslant\left(\int_{K_{R} \cap\{\rho<|x|<2 \rho\}} u^{l} d x\right)^{1 / l}\left(\frac{C}{\rho^{\left(2-s_{*}\right) l^{\prime}-N}}\right)^{1 / l^{\prime}} .
\end{aligned}
$$

Первый интеграл стремится к нулю при $\rho \rightarrow \infty$ (как в предельном случае $q=q^{*}$ теоремы 1$)$, поэтому при $\left(2-s_{*}\right) l^{\prime}-N \geqslant 0$ имеем отсутствие нетривиального решения для всех $q \in \mathbb{R}$.

Полученный критический показатель $l^{*}$ является неулучшаемым. Действительно, рассмотрим функцию

$$
u(x) \equiv u(r, \omega)=\varepsilon r^{s} \Phi(\omega),
$$


где $\varepsilon>0$. Как уже вычислялось ранее,

$$
-\Delta u=-\varepsilon \Phi r^{s-2}\left\{s^{2}+s(N-2)-\lambda_{\omega}\right\} .
$$

Выберем $s<0$ такое, что $s^{2}+s(N-2)-\lambda_{\omega}=-\delta<0$, и положим $\varepsilon \delta=\varepsilon^{q}$. Считая для простоты, что $R>1$ (т.е. в нашей области $K_{R}$ имеем $|x|=r>1$ ) и $q>0$, можем использовать неравенство $r^{s-2} \geqslant r^{s q}$ при $s-2 \geqslant s q($ так как $s<0)$. Тогда

$$
-\Delta u=\varepsilon^{q} r^{s-2} \Phi \geqslant\left(\varepsilon r^{s} \Phi\right)^{q}=u^{q} .
$$

Условия на $s$ вместе с требованием суммируемости $u(x) \in L_{l}\left(K_{R}\right)$ дают

$$
\left\{\begin{array}{l}
s-2 \geqslant s q \\
-s^{*}<s<0 \\
s l<-N .
\end{array}\right.
$$

Выполнение этих условий следует из

что, в свою очередь, вытекает из

$$
\left\{\begin{array}{l}
-s^{*}<s \leqslant-\frac{2}{q-1} \\
-s^{*}<s<-\frac{N}{l}
\end{array}\right.
$$

$$
\left\{\begin{array}{l}
q>1+\frac{2}{s^{*}}, \\
l>\frac{N}{s^{*}} .
\end{array}\right.
$$

Первое из этих неравенств отражает критический показатель отсутствия решений без условия на бесконечности, а второе - наше требование суммируемости.

Поскольку мы рассматриваем коническую область, то естественно обобщить эту теорему 3 на пространства типа Кондратьева со степенным весом.

TeOpema 4. Пусть $\alpha \in \mathbb{R} u$

$$
1<l \leqslant l^{*}=\frac{N}{s^{*}-\alpha} .
$$

Тогда задача (1) ни при каких $q \in \mathbb{R}$ не имеет слабого решения $u(x)$ такого, что

$$
u(x)|x|^{\alpha} \in L_{l}\left(K_{R}\right) .
$$

ДокАЗАТЕльСтво. Аналогично предыдущему доказательству требуемое утверждение следует из оценки

$$
\begin{aligned}
\int_{K_{R}} u^{q} \varphi_{\rho} d x & \leqslant \int_{K_{R} \cap\{\rho<|x|<2 \rho\}} u|x|^{\alpha} \frac{\left|\Delta \varphi_{\rho}\right|}{|x|^{\alpha}} d x \\
& \leqslant\left(\int_{K_{R} \cap\{\rho<|x|<2 \rho\}}\left(u|x|^{\alpha}\right)^{l} d x\right)^{1 / l}\left(\int_{K_{R} \cap\{\rho<|x|<2 \rho\}} \frac{\left|\Delta \varphi_{\rho}\right|^{l^{\prime}}}{|x|^{\alpha l^{\prime}}} d x\right)^{1 / l^{\prime}} \\
& \leqslant\left(\int_{K_{R} \cap\{\rho<|x|<2 \rho\}}\left(u|x|^{\alpha}\right)^{l} d x\right)^{1 / l}\left(\frac{C}{\rho^{\left(\alpha+2-s_{*}\right) l^{\prime}-N}}\right)^{1 / l^{\prime}}
\end{aligned}
$$

В заключение автор выражает благодарность С. И. Похожаеву за постановку задачи и В.В. Курте за полезное обсуждение результатов. 


\section{СПИСОК ЦИТИРОВАННОЙ ЛИТЕРАТУРЫ}

[1] Кондратьев В.А. Краевые задачи для эллиптических уравнений в областях с коническими и угловыми точками // Тр. ММО. 1967. Т. 16. С. 209-292.

[2] Назаров С. А., Пламеневский Б. А. Эллиптические задачи в областях с кусочно гладкой границей. М.: Наука, 1991.

[3] Egnell H. Positive solutions of semilinear equations in cones // Trans. Amer. Math. Soc. 1992. V. 330. P. 191-201.

[4] Нгуен Мань Хунг. Об отсутствии положительных решений нелинейных эллиптических уравнений второго порядка в конических областях // Дифференц. уравнения. 1998. Т. 34. C. $533-539$.

[5] Коньков А. А. О неотрицательных решениях квазилинейных эллиптических неравенств // Изв. РАН. Сер. матем. 1999. Т. 63. С. 41-127.

[6] Кондратьев В.А., Ландис Е. М. О качественных свойствах решений одного нелинейного уравнения второго порядка // Матем. сб. 1988. Т. 135. №6. С. 346-360.

[7] Похожаев С. И. Существенно нелинейные емкости, индуцированные дифференциальными операторами // Докл. РАН. 1997. Т. 357. С. 592-594.

[8] Митидиери Э., Похожаев С. И. Отсутствие глобальных положительных решений квазилинейных эллиптических неравенств // Докл. РАН. 1998. Т. 359. С. 456-460.

[9] Митидиери Э., Похожаев С.И.Отсутствие положительных решений для квазилинейных эллиптических задач в $\mathbb{R}^{N} / /$ Тр. МИАН. 1999. Т. 227. С. 192-222.

[10] Курта В. В. Некоторые вопросы качественной теории нелинейных дифференциальных уравнений второго порядка. Дисс. ... д. ф.-м. н. М.: МИАН, 1994.

[11] Курта В.В.К вопросу об отсутствии целых положительных решений у полулинейных эллиптических уравнений // УМН. 1995. Т. 50. №4. С. 131.

[12] Курта В.В. Об отсутствии положительных решений у полулинейных эллиптических уравнений // Тр. МИАН. 1999. Т. 227. С. 162-169.

[13] Митидиери Э., Похожаев С. И. Априорные оценки и отсутствие решений нелинейных уравнений и неравенств в частных производных. Тр. МИАН. Т. 234. М.: Наука, 2001.

[14] Bandle C., Essen M. On positive solutions of Emden equations in cone-like domains // Arch. Rational Mech. Anal. 1990. V. 112. P. 319-338.

[15] Bandle C. Positive solutions of Emden equations in a cone-like domains // Progress in Nonlinear Differential Equations Appl. 1992. V. 7. P. 71-75.

[16] Лаптев Г. Г. Отсутствие глобальных положительных решений систем полулинейных эллиптических неравенств в конусах // Изв. РАН. Сер. матем. 2000. Т. 64. № 6. С. 107-124.

[17] Laptev G. G. Some nonexistence results for higher-order evolution inequalities in cone-like domains // Electron. Res. Announc. Amer. Math. Soc. 2001. V. 7. P. 87-93.

[18] Bidaut-Veron M.-F., Pohozaev S. Nonexistence results and estimates for some nonlinear elliptic problems // J. Anal. Math. 2001. V. 84. P. 1-49.

[19] Kartsatos A. G., Kurta V. V. Nonexistence theorems for weak solutions of quasilinear elliptic equations // Abstract Appl. Anal. 2000. №6. P. 163-189.

[20] Brezis H., Cabré X. Some simple nonlinear PDE's without solutions // Boll. Unione Mat. Ital. Sez. B Artic. Ric. Mat. (8). 1998. V. 1. P. 223-262.

[21] Galakhov E. Some nonexistence results for quasilinear elliptic problems // J. Math. Anal. Appl. 2000. V. 252. P. 256-277.

[22] Похожаев С. И., Тесей А. Полное разрушение решений нелинейных эллиптических неравенств // Дифференц. уравнения. 2001. Т. 37. С. 521-528.

[23] Лаптев Г. Г. Об отсутствии решений одного класса сингулярных полулинейных дифференциальных неравенств // Тр. МИАН. 2001. Т. 232. С. 223-235.

[24] Митидиери Э., Похожаев С.И. Некоторые обобщения теоремы Бернштейна // Дифференц. уравнения. 2002. Т. 38 (в печати). 